\title{
BRAsile, la STElla DEL Sud
}

\section{Paolo Spedicato}

RESENHA Brasile, la stella del Sud

Quaderni Speciali di Limes. Supplemento al n. 3/2007.

Direttore responsabile Lucio Caracciolo.

Roma, Gruppo Editoriale L’Espresso spa.

www.limesonline.com 


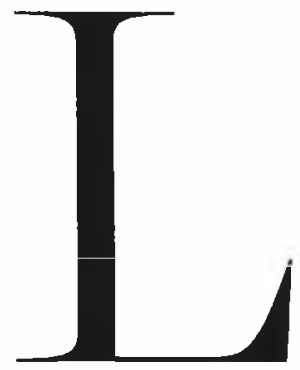

a recente pubblicazione nei Quaderni Speciali di Limes, prestigiosa rivista italiana di geopolitica, di un numero unico su Brasile, la stella del sud, è certamente segno dei tempi e frutto di un nuovo interesse politico-culturale dell'Italia contemporanea verso il Brasile e l'America Latina. Il numero è articolato in tre parti. La prima, "É nata una stella”, di contenuto storico e geopolitico propriamente detto, una seconda, "Fratture e frontiere nel continente verdeoro", sulle prospettive identitarie, tra natura, cultura e vocazione industriale, tra presente problematico e futuro promettente. Chiude il numero una terza parte, "Italia-Brasile, andata e ritorno", che aggiorna su un rapporto ritrovato, dopo l'indifferenza degli anni '90-inizio 2000,e sul quale qui si vuole spendere qualche riflessione.

L'intervento dell'Ambasciatore Ludovico Incisa di Camerana, autore di una ricostruzione storica, Il grande esodo (Milano: Corbaccio, 2003), offre l'opportunità di riconsiderare le diverse fasi storiche della experience italiana, direbbero gli americani, a San Paolo, forse la città più italiana del mondo, vista dall'ottica dei flussi emigratori di massa a partire dagli anni '70 del secolo XIX. A partire dalla "novità" per il Brasile dell'inserimento nella realtà lavorativa di ragazzi e donne, alla costituzione di veri e propri quartieri urbani costruiti a ridosso delle fabbriche e delle attività edilizie ("alla fine dell'Ottocento a San Paolo due terzi dei muratori e la maggioranza assoluta dei capi mastri erano italiani"), fino all'organizzazione del lavoro sfruttato nelle fazendas ${ }^{1}$ di caffè nell'interno dello stato, l'analisi di Incisa prosegue con l'apporto fondamentale dato dall'arrivo dall'Italia di una classe di alto borghesi, protagonisti della rivoluzione industriale e commerciale della città e dello stato: "I nuovi venuti - i Matarazzo, i Crespi, i Pinotti Gamba, i Siciliano, gli Scarpa, e più tardi i Ramenzoni e i Martinelli - entreranno nella classe dirigente e si serviranno di maestranze italiane". Di questa 
lunga marcia degli italiani a San Paolo e non solo fa parte un incidente politico-diplomatico tra Italia e Brasile, a seguito della sconfitta italiana di Adua in Etiopia (1 marzo 1896) e dell'appannarsi del progetto imperiale dell'I talia in Africa, voluto prima da Crispi e poi da Giolitti, i cui governi furono notoriamente contraddittori e ambigui in materia di emigrazione, che continuava inarrestabile. Il contemporaneo non sopito secessionismo degli stati brasiliani del sud con il coinvolgimento di residenti italiani, una conseguente crisi militare-diplomatica tra i due paesi, l'attivismo mal tollerato del console italiano a San Paolo, il savoiardo Compans de Brichanteau, costituiscono un brutto momento per le nostre comunità, forse comparabile con le conseguenze del voltafaccia vargasiano contro il fenomeno integralista all'epoca dell'asse Roma-Berlino, e la repressione della lingua e di ogni vestigio di identità italiana nelle comunità di oriundi.

Ma è sul terreno della cultura di avanguardia che la modernità italiana e brasiliana finiscono per riincontrarsi. Filippo Tommaso Marinetti ha lanciato sulle pagine de Le Figaro nel 1909 il Manifesto del Futurismo, prima avanguardia storica del secolo. La risposta brasiliana arriverà con la Semana Modernista paulista del '22 e con la conseguente amicizia tra Marinetti e l'industrialegiornalista Assis Chateaubriand, che l'italiano, in una visita a Rio nel 1926, definirà "unico futurista sudamericano". È durante questa visita carioca, menzionata da Incisa, che Marinetti, accompagnato da Chateaubriand e dallo scrittore Rodrigo de Melo Franco, visitò il Morro do Pinto, sotto scorta poliziesca. Scrisse un cronista dell'epoca: "Marinetti recebera do morro informações erradas. Bam-bam-bans de borrachas brancas, navalha no bolso, esperando 'um risco' pra barriga, os amores beirando sempre a morte. Ao contrário, encontrou a favela pacata. Feliz" ${ }^{2}$. Traluci e ombre ricorrenti nel rapporto tra le due identità, e tra italiani stessi divisi tra borghesia imprenditoriale e italianini del proletariato industriale, Incisa conclude che "La scelta dell'invisibilità etnica adottata da molti imprenditori italiani sarà un fenomeno di lunga durata e comune agli italiani ed ex italiani residenti in America Latina, ma più diffuso in Brasile... Il timore di un'eccessiva visibilità politica si accentuerà peraltro in Brasile durante la seconda guerra mondiale”. Un'altra conseguenza di queste dinamiche sembra essere stata una regola di divisione del lavoro: "ai brasiliani il potere politico, agli italiani e agli altri stranieri l'industria"

La metropoli paulista continua a svolgere un ruolo centrale nel paese e negli equilibri della mondializzazione. L'italianità storico-culturale di San Paolo è ancora presente, aggiornata, in questo cammino di metropoli-stato protagonista. L'industria culturale e attività a loro modo parallele come la moda, il design, il cinema, l'architettura, hanno ricevuto grande impulso dalla nostra penisola. A cominciare negli anni '30 del secolo scorso dagli architetti Pietro Maria Bardi e Lina Bo Bardi, all'insegnamento di Giuseppe Ungaretti alla USP, alle incursioni nel cinema brasiliano di Adolfo Celi, Luciano Salce e Gianni Ratto, nel teatro di Gianfrancesco Guarnieri, nel giornalismo di Mino Carta, attuale direttore del settimanale Carta Capital, dell'oriundo Claudio Lachini, ex amministratore delegato del giornale Gazeta Mercantil, del polemista della rivista Veja, l'altro oriundo Diogo Mainardi, di José R. Guzzo della Editora Abril, dello storico Elio Gaspari, di Carlos 
Marchi dell'Estado de São Paulo, di Roberto Mugiatti ex direttore della rivista Manchete, del compianto Claudio Abramo ex direttore della Folha de São Paulo. Le case editrici diSan Paolo, e di Rio, sono attentissime a proporre i classici e i contemporanei della nostra letteratura, filosofia, diritto, politologia: i filosofi Bobbio, Vattimo, Agamben, Negri, come i classici delle nostre lettere moderne Verga, Pirandello, Pavese o romanzieri come Sciascia, Tabucchi, Camilleri, Baricco. Basti accennare alle case editrici Cosac \& Naify, Ateliê Editora, Berlendis \& Vertecchia, Barcarolla, Rocco...

Ma esiste un lascito di italianità così evidente e non sufficientemente evidenziato, lì a disposizione per chi passa per le strade, le piazze, i palazzi storici di San Paolo. É l'impronta lasciata dagli artisti, scultori, decoratori italiani, da maestranze e artigiani delle arti visive attraverso i decenni. Nel 1993 il Consolato Generale d'Italia diSan Paolo pubblicò un catalogo bilingue a cura del fotografo paulista Bruno Giovannetti dedicato appunto a Artisti italiani nelle piazze di San Paolo. E come dimenticare il contributo degli architetti militari toscani, genovesi, mantovani che costruirono attraverso i secoli piazzeforti e fortezze lungo la costa oceanica e i fiumi brasiliani, dal litorale paulista fino alle coste del remoto Amapá, gli stessi che si spinsero a edificare tra la Colombia, Cuba e Portorico?

Sempre all'interno della stessa sezione "Italia-Brasile. Andata e ritorno", l'attuale Ambasciatore italiano a Brasilia, Michele Valensise, si misura con il supposto carattere nazionale dei brasiliani, all'insegna del famoso "jeitinho, parola intraducibile che indica, più che un metodo, una categoria dello spirito. É la capacità - continua Valensise - di trovare una soluzione soddisfacente a un problema, la via d'uscita migliore da una situazione di stallo, aggirando a buon fine la rigidità di norme e convenzioni. Si superano cosi impedimenti e ostacoli di ogni tipo, legislativo, burocratico, personale. Ė il trionfo della flessibilità e dell'inventiva”. La stessa storia del Brasile moderno sarebbe il frutto di questa disposizione al compromesso e alla mediazione, contraria quindi alla contrapposizione dialettica e alle svolte radicali. Secondo Valensise l'Italia di oggi dovrebbe approfittare di queste "affinità psicologiche e culturali sviluppatesi più di quanto si possa immaginare nell'arco di decenni di interazione"

Vale la pena però approfondire una questione non così pacifica, come l'Ambasciatore sembra pensare. D'accordo, la questione può essere discussa a partire dall'idea di "uomo cordiale", concetto inventato dal poeta modernista Ribeiro Couto e affrontata nel grande libro di Sérgio Buarque de Holanda Raizes do Brasil (1936), come lo stesso Valensise ricorda. Ma lo stesso Buarque era alquanto cauteloso e critico a proposito di cordialità brasiliana, non tralasciando di metterla in relazione con una specifica mentalità iberica (e probabilmente di tutta l'Europa del Sud), fatta di spirito di clan, di anacronistica promiscuità con i parenti prossimi e i socialmente affini, di comportamenti da hidalgos di provincia che aborrono il lavoro e lo demandano ai sottoposti. Raízes do Brasil del resto era già un'analisi weberiana del Brasile e un libro "tedesco", frutto di un periodo di studio di quattro anni in Germania.

Questa quasi naturale virtù nazionale entra anche nel discorso politico brasiliano, secondo l'articolista. Ne è riprova il recente ritorno, come senatore federale eletto, del politico più contro- 
verso nella storia della giovane democrazia brasiliana, l'ex presidente della Repubblica, l'alagoano Fernando Collor de Mello, cacciato nel 1992 a furor di popolo da milioni di caras pintadas. Così Valensise riassume la vicenda: "Ora in Senato ha pronunciato il suo primo discorso, applaudito da destra a sinistra. Tutti d'accordo, senza rancori. Tudo bem". Si ha l'impressione di sentire l'eco di altri stereotipi paralleli, è questa la parola giusta, e non proprio esaltanti, del tipo: "tarallucci e vino", "italiani brava gente" "tengo famiglia" e simili. In sostanza, ci sembra che siffatta ricostruzione storica sia insufficiente e l'interpretazione politica limitata e a livello di un esotismo di comodo, oltre che moralmente discutibile. Innanzitutto la faccenda del jeitinho non è nemmeno di gradimento universale. Lo ricordava, in margine a una recente inchiesta del quotidiano $O$ Globo sull'impunità, "O Brasil vive o crime sem castigo", l'economista della Fondazione Getúlio Vargas, Marcos Fernandes: “Na França, um processo é analisado ejulgado em três meses; no Brasil, em dez anos. Impunidade, por que ela existe? Não há lei no Brasil, simples. As pessoas se prendem abanali.dades preconceituosas do tipo ‘o brasileiro é cheio de jeitinho' ou 'aqui o que vale é a lei de Gerson'. Um francês não é diferente de um brasileiro (até onde sei, as leis da genética não foram violadas): o problema está nas instituições. Reformas já: reformas do Judiciário, política e administrativa" (23 giugno 2007, p. 4). Sempre all'interno della stessa inchiesta il giornale carioca aveva pubblicato il giorno prima (p. 4) l'intervista a Jorge Bandeira de Mello, ex socio e ex pilota di aereo di P.C. Farias, tesoriere corrotto del deposto presidente, trovato morto assassinato in una stanza d'albergo. Una frase era illuminante: "Tenho muitos amigos em todos os partidos. Em Alagoas, há uma família só". Viene in mente un altro detto, analogo ma esecrabile, brasiliano: "Aos amigos tudo, aos inimigos a lei". Non può essere considerata innocente questa filosofia spicciola del Tudo bem, Tudo jóia, del Se dar muito bem, soprattutto se applicata ai rapporti politico-sociali. Su questa falsariga vengono in mente utilmente, specie in un'epoca di rimozione collettiva del passato in Brasile come in Italia, fatti e fenomeni che hanno caratterizzato la nostra storia nazionale e che, se non possono essere automaticamente tradotti in maschere nazionali, vanno però pensati come avvertimenti seriper la memoria e il costume del nostro paese. Vogliamo provare a ricordarne qualcuno? Il trasformismo politico inaugurato dai governi della liberale "Sinistra storica" (1876-87) all'indomani del Risorgimento; il "familismo [più o meno, N.d.A.] amorale", secondo la nota e controversa tesi del sociologo americano Edward Banfield, individuato nella società agricola di un paesino lucano a metà anni '50, e basato sui valori della famiglia mononucleare tutta chiusa al sociale e al bene comune; la struttura economico-corporativa della penisola durante secoli (con l'aggiornamento del neolobbismo e neocorporativismo odierni); gli eccessivi perdonismo e pentitismo di matrice cattolica...

La pubblicazione del quaderno brasiliano di Limes è occasione importante per riflettere sul rafforzamento delle relazioni tra Italia e Brasile, paesi che si vorrebbe vedere come protagonisti di una mondializzazione responsabile e della crescente "domanda di politica estera", ben al di là di vecchi stereotipi e di pratiche moralmente e politicamente obsolete. 